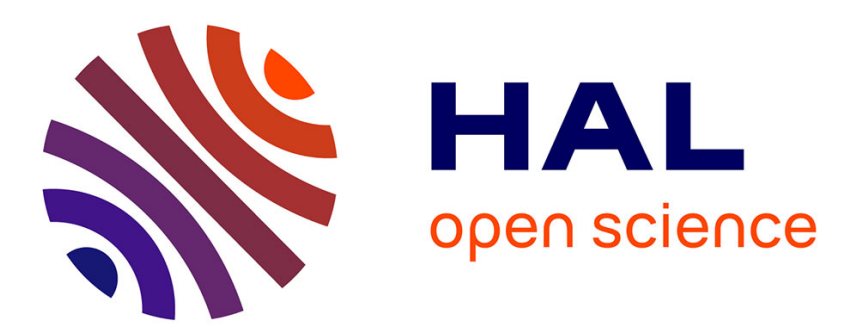

\title{
Réflexion cohérente et incohérente d'une onde acoustique plane harmonique par un rideau de bulles stratifié plan \\ F. Delaveau, M. Gatulle
}

\section{- To cite this version:}

F. Delaveau, M. Gatulle. Réflexion cohérente et incohérente d'une onde acoustique plane harmonique par un rideau de bulles stratifié plan. Journal de Physique IV Proceedings, 1994, 04 (C5), pp.C51141-C5-1144. 10.1051/jp4:19945252 . jpa-00252941

\section{HAL Id: jpa-00252941 https://hal.science/jpa-00252941}

Submitted on 1 Jan 1994

HAL is a multi-disciplinary open access archive for the deposit and dissemination of scientific research documents, whether they are published or not. The documents may come from teaching and research institutions in France or abroad, or from public or private research centers.
L'archive ouverte pluridisciplinaire HAL, est destinée au dépôt et à la diffusion de documents scientifiques de niveau recherche, publiés ou non, émanant des établissements d'enseignement et de recherche français ou étrangers, des laboratoires publics ou privés. 


\title{
Réflexion cohérente et incohérente d'une onde acoustique plane harmonique par un rideau de bulles stratifié plan
}

\author{
F. DELAVEAU et M. GATULLE*
}

Société AERO, 3 avenue de l'Opéra, 75001 Paris, France
${ }^{*}$ Commissariat à l'Energie Atomique, Centre de Vaujours, BP. 7, 77181 Courtry, France

\begin{abstract}
Résumé : A mathematical and numerical model is presented for the prediction of the coherent and incoherent acoustic reflexion coefficients for bubble layers in a liquid medium, excited by a harmonic pressure farfield. The layer is plane infinite, and looks like a random stratified system, where bubble position and size obey a statistical distribution, that depends on immersion only. The bubbles are assumed to be spherical at rest. No hypothesis is made about the layer's thickness, neither on the process of bubbles interaction and scattering. All linear bubble deformations and multiple scattering are considered, as well as thermal conduction in the bubbles. The geometrical configuration leads us to use a mathematical transformation of the acoustic integral equation, that "delocalizes" the observation in a plane parallel to the layer and introduces natural collective contributions of bubbles at each immersion, without any physical approximation. After this transformation, coherent and incoherent acoustic pressure are obtained in the form of an integral equation over the thickness of the layer. We present the general model, the mathematical formulation and numerical results. We show the link between our results and Foldy's formalism, when only monopolar diffusion is considered.
\end{abstract}

\section{INTRODUCTION}

On sait depuis plus d'un siècle que la présence de bulles de gaz en milieu liquide perturbe de manière importante la propagation des ondes acoustiques. L'étude de la propagation des ondes acoustiques en milieu diphasique a suscité de nombreuses recherches et expérimentations ([1], [2], [3], [4], [5], [6], [7], [8], [11] etc.). Une méthode souvent utilisée consiste à rechercher des propriétés locales cohérentes équivalentes du milieu inhomogène supposé infini (voir par exemple [6],[7]). La démarche effectuée ici est radicalement différente, et utilise l'invariance statistique par translation parallèle à un rideau stratifié horizontalement, pour obtenir les composantes cohérente et incohérente du champ diffusé $\left(^{1}\right)$.

Les bulles sont situées dans un rideau plan, d'extension latérale infinie et d'épaisseur h donnée (voir figure 1) de densité volumique quelconque (sauf pour certaines simplifications dans le calcul du champ incohérent). On les suppose sphériques au repos, leur déformation dynamique étant linéaire quelconque. Leur densité, leurs positions et leurs rayons sont des quantités aléatoires de loi de probabilité donnée, fonctions de la seule immersion $z$. La dynamique de chaque bulle est décrite de manière équivalente à celle employée par exemple en [1], elle tient compte des échanges de chaleur dans le gaz (analyse thermodynamique complète) et de la tension superficielle à l'interface entre le gaz et le liquide ambiant. On utilise ainsi l'équation de continuité, l'équation d'Euler, une équation d'état et une équation thermique linéarisées pour chaque milieu, ainsi que l'équation de Laplace pour le raccordement à l'interface eau/gaz. La modélisation ignore les effets visqueux, qui pourraient être introduits sans difficulté majeure. Le rideau est insoné par une onde acoustique plane harmonique à la pulsation $\omega$ (onde d'extension infinie) d'incidence quelconque (cf figure 1). Il est supposé entièrement immergé (l'oubli d'un éventuel dioptre air/liquide n'est pas déterminant dans l'élaboration du modèle).

\footnotetext{
1 Le champ cohérent (resp. incohérent) est la moyenne (resp. l'écart type) du champ aléaloire de pression. Le champ cohérent contient les effets de phase entre les contributions des diffuseurs aléatoirement répartis.
} 


\section{DIFFUSION D'UNE BULLE}

Les grandeurs physiques externes (i.e dans le fluide) et internes (i.e dans le gaz), ainsi que le déplacement normal à la surface de chaque bulle sont décomposés en harmoniques sphériques centrées sur la bulle : ceci conduit par exemple pour la pression à une écriture du type $P_{l}(\vec{r})-P_{0}=\sum_{n \geq 0} \sum_{m=-n}^{n} p_{n}^{m}(l) . X_{n}^{m}(\vec{r}), \mathrm{P}_{0}$ étant la pression à l'équilibre, $\vec{r}$ le vecteur entre le point d'observation $\mathrm{M}$ et le centre de la bulle d'indice 1 , et $X_{n}^{m}(\vec{r})$ l'harmonique sphérique d'indices n,m (cf [9]). Après élimination des composantes sphériques de la masse volumique et de la température dans chaque fluide, on montre que les composantes sphériques de la pression interne (resp. externe) et de la vitesse normale sur chaque bulle sont reliées par un coefficient de transfert multipolaire "interne" i $\beta_{n}$ (resp. "externe" e $\beta_{n}$ ). Ces deux relations de transfert contiennent toute linformation sur la dynamique intrinsèque de la bulle $\left({ }^{2}\right)$. Elles s'écrivent $\left({ }^{3}\right)$ :

$$
\begin{aligned}
& \text { pour }-n \leq m \leq n \quad \frac{{ }^{i} \beta_{n}}{Z_{2}}=\frac{u_{n}^{m}(a)}{i p_{n}^{m}(a)}, \frac{{ }^{e} \beta_{n}(a)}{Z_{2}}=\frac{u_{n}^{m}(a)}{{ }^{e} p_{n}^{m}(a)} \text { (1) avec : } \\
& { }^{i} \beta_{n}=\frac{\left(\gamma_{2}-1\right) \chi_{2} \omega}{c_{2}{ }^{2}}\left[\frac{x_{q}}{x_{1}} \frac{j_{n+1}\left(x_{q}\right)}{j_{n}\left(x_{q}\right)}+i\left(\frac{x_{1}}{2 n+3}-\frac{n}{x_{2}}\right)\right] \quad \text { (2) }{ }^{e} \beta_{n}(a)=\frac{{ }^{i} \beta_{n}}{1-[2-n(n+1)]{ }^{i} \beta_{n} \sigma /\left(i \omega a^{2} Z_{2}\right)}
\end{aligned}
$$

\section{DIFFUSION DU RIDEAU STRATIFIE}

En utilisant les équations intégrales classiques de l'acoustique harmonique, on montre alors que le champ de pression en tout point est caractérisé par les valeurs des composantes sphériques externes ${ }^{e} p_{n}^{m}(l)\left({ }^{4}\right)$ :

$$
P_{d}\left(\vec{R}_{0}\right)=\sum_{\text {bulles }} \sum_{\substack{n \geq 0 \\ m=-n, n}} x_{1}^{2}(l){ }^{3} Z_{n}^{m}\left(k_{1} \vec{r}_{l}\right){ }^{e} p_{n}^{m}(l)\left[i j_{n}\left(x_{1}(l)\right)+\frac{Z_{1}}{Z_{2}}{ }^{e} \beta_{n}^{m}(l) j_{n}\left(x_{1}(l)\right)\right]
$$

En faisant tendre le point d'observation $M_{0}$ vers chaque bulle $I_{0}$ du rideau, on obtient à l'aide de l'expression ci-dessus un système linéaire (infini) vérifié par les ${ }^{c} p_{n}^{m}(l)$. Il est écrit ci-dessous sous une forme condensée (pour toute bulle $l_{0}$ et tout couples $(n, m) ; n \geq 0,-n \leq m \leq n$ ) :

$\sum_{\text {bulles } l} \sum_{\substack{p \geq 0, q=-p, p}} M_{n p}^{m q}\left(l, l_{0}\right)^{e} p_{p}^{q}(l)=p_{0} S_{n}^{m}\left(l_{0}\right)$

$M_{n p}^{m a}\left(l, l_{0}\right)$ contient le coefficient de transfert ${ }^{\mathrm{e}} \beta_{\mathrm{n}}(1)$ de la bulle 1 , les propriétés diffusantes des bulles 1 et $l_{0}$, ainsi que leurs interactions, et rend compte des phénomènes de diffusion multiple, tandis que $S_{n}^{m}\left(l_{0}\right)$ fait intervenir les propriétés diffusantes de la bulle $l_{0}$.

\section{TRAITEMENT STATISTIQUE}

Ce traitement donne accès aux champs cohérents et incohérents réfléchis. La méthode met à profit une hypothèse d'ergodicité qui peut s'énoncer ainsi : "Considérer toutes les configurations possibles des bulles (position et taille) en un point $M$ fixé d' un plan $\Pi$ (extérieur au rideau et parallèle à celui-ci) équivaut à observer une configuration donnée des bulles dans le rideau à partir de tous les points $M$ du plan $\Pi^{\prime}$.

\footnotetext{
${ }^{2}$ Dans [1], Prosperetui considère la seule diffusion monopolaire (les bulles vibrent en restant sphériques). Si l'on ne retient que le transfert monopolaire $(m=n=0)$, la modélisation adoptéc ici est tout à fait équivalente à la viscosité près

${ }^{3} \mathrm{~L}$ 'indice $l$ se rapporte à la bulle (de rayon $\mathrm{a}_{1}$ ), l'indicc 1 au fluide extérieur, l'indice 2 au ga $z, c_{1}$ et $c_{2}$ désignent la célérité du son dans l'eau et dans le gaz, $\vec{k}_{1}$ désigne le nombre d'onde incident; $k_{1}=\omega / c_{1}$ son module, $k_{2}=\omega / c_{2}$ le module du nombre d'onde dans le gaz. $j_{n}$ désigne la fonction de Bessel sphérique dordre $n . \sigma$ désigne la tension superficielle, $\omega$ la pulsation, a le rayon de la bulle considérée, $Z_{1}$ et $Z_{2}$ sont les impédances acoustiques (adiabatiques) du liquide et du gaz : $x_{1}=k_{1} a, x_{2}=k_{2} a$, $x_{q}=k_{q} a$, avec $k_{q}=e^{i \pi / 4}\left(\omega / \chi_{2}\right)^{1 / 2} \cdot x_{2}$ et $\gamma_{2}=C_{p} / C_{v}$ se rapportent au gaz et sont respectivement la diffusivité thermique et le rapport des capacités calorifiques à pression et volume constant.

$4^{3} Z^{m}{ }_{n}$ désigne l'harmonique sphérique scalairc ondulatoire de Hankel première espèce (voir [9]), $\vec{R}_{0}$ désigne le vecteur entre le point d'origine 0 et le point d'observation $M_{0}$. Toutes les autres quantités se rapportent à la bulle $l: \vec{r}_{l}$ désigne le vecteur entre le centre de la bulle $l$ et le point d'observation $M_{0}$, al le rayon de la bulle $l$, et $x_{1}(l)=k_{1} a_{l}$ elc.
} 
On discrétise ensuite le rideau en couches d'épaisseur infinitésimale $\delta$ h puis on réarrange formellement les bulles pour faire apparaître une réaction du rideau couche par couche, et non plus bulle par bulle. De plus, cette hypothèse d'ergodicité permet d'accéder par des intégrations spatiales aux moments d'ordre 1 et 2 des grandeurs aléatoires qui traduisent le comportement statistique collectif des "bulles d'une même couche". Chaque composante de Fourier bidimensionnelle de la pression diffusée sur le plan d'observation $\Pi_{0}$ s'écrit alors sous la forme $P_{d}\left(\overrightarrow{\mathbf{K}}, M_{o}\right)=4 . \pi^{2} P_{d c}\left(\vec{k}_{1 x}, z_{o}\right) \delta\left(\overrightarrow{\mathbf{K}}-\vec{k}_{1 x}\right)+D\left(\overrightarrow{\mathbf{K}}, z_{o}\right)\left(\vec{k}_{1 x}\right.$ est la projection de $\vec{k}_{1}$ sur $\Pi_{0}, \overrightarrow{\mathbf{K}}$ est la variable de Fourier parrallèle à $\Pi_{0}$ - voir figure 1$)$. Cette écriture, classique dans l'étude de la réflexion par des structures planes aléatoires, traduit d'une part la cristallisation d'une partie de l'énergie réfléchie sur la composante spectrale $\mathrm{P}_{\mathrm{dc}}\left(\vec{k}_{1 x}, \mathrm{M}_{\mathrm{O}}\right)$ (composante cohérente), conformément aux lois de Descartes, et d'autre part l'existence d'une énergie diffuse répartie continûment sur toutes les composantes du spectre spatial (composante incohérente). La formulation (4) permet alors d'exprimer ses composantes de Fourier bidimensionnelles du champ de pression lointain sous la forme de l'intégrale sur l'épaisseur du rideau d'une fonction auxiliaire inconnue $A(z)$ vérifiant elle-même une équation intégrale sur l'immersion $z$, obtenue à partir du système linéaire (5) et des réarrangements des bulles mentionnés plus haut, dans laquelle interviennent des noyaux où apparaissent les propriétés diffusantes moyennes des bulles situées à la cote $\mathrm{z}$, notamment la transmission et la rétrodiffusion des bulles d'une même couche.

Les expressions finales, complexes dans le cas général, se prétent à une analyse physique pour la contribution monopolaire des bulles, prépondérante dans nos applications (le taux d'air étant faible). On obtient en effet les coefficients de réflexion cohérent et incohérent $\left({ }^{5}\right)$ sous la forme suivante $\left(^{6}\right)$ :

$$
R_{c}=\left|\frac{1}{2 \pi k_{1} k_{1 z}} \int_{z=0}^{n} e^{i k_{12}(z)} \cdot A(z) d z\right|^{2} \quad \text { (6), } \quad R_{i}=\frac{1}{(2 \pi)^{3} k_{1} k_{1 z}} \int_{z=0}^{h} \frac{\left\langle|\alpha(z)|^{2}\right\rangle}{n(z)|\langle\alpha(z)\rangle|^{2}} \cdot|A(z)|^{2} d z
$$

Dans le cas pratique où les propriétés diffusantes des couches sont lentement variables avec l'immersion (devant le champ lui-même, qui est très rapidement atténué), $A(z)$ et le champ de pression cohérent vérifient une équation différencielle du type Hemholtz avec un nombre d'ondes équivalent identique à celui obtenu par Foldy [6]. Enfin, la formule (7) montre que dans le cas de contributions monopolaires seules, la contribution incohérente au champ diffusé par une bulle dépend de la seule excitation cohérente. La résolution est ensuite numérique.

\section{PRINCIPAUX RESULTATS:}

La réflexion cohérente obtenue avec les contributions multipolaires est numériquement identique à celle que donne le formalisme de Foldy (théorie de milieu effectif), malgré les hypothèses restrictives inhérentes à l'élaboration de ce dernier [7]. Ce point a été confirmé analytiquement lorsque seule la diffusion monopolaire est prise en compte. Il semble donc que les contributions aux ordres $n \geq 1$ (qui sont en bande étroite) puissent être négligées: même avec de forte densités de bulles, les monopoles seuls suffisent à rendre compte de la réflexion cohérente quasi-totale (leurs effers en bande large s'accroissent avec le taux d'air).

Les coefficients de réflexions cohérent et incohérent sont finis. Par ailleurs, la variance du champ de pression a été évaluée et présente une dépendance logarithmique en fonction de la surface insonée.

Le rapport entre énergie cohérente et énergie incohérente réfléchies est fonction de la fréquence et de l'angle d'insonation : la comparaison d'un modèle théorique avec des résultats expérimentaux (par exemple ceux figurant en [8]) doit donc être effectuée avec précautions.

\section{CONCLUSIONS - PERSPECTIVES}

Le point clé du présent modèle est l'invariance statistique de la répartition des diffuseurs au moins localement sur une direction. Il peut être étendu à des configurations plus réalistes:

. introduction d'un dioptre (même rugueux),

\footnotetext{
5 On montre en écrivant le vecteur de Poynting que l'énergic tolale diffuscé par le rideau. traversant une surface unité $S\left(z_{0}\right)$ à la cote $z_{0}$, est la somme de deux contributions, l'une cohérente et l'autre incohérente. Le coefficient de réflexion cohérent (resp. incohérent) est défini comme le rapport entre l'énergie cohérente (resp. incohérente) et l'énergie incidente traversant $S\left(z_{1}\right)$ ).

$6 \mathrm{n}(\mathrm{z})$ est la dersité des bulles à la cote $z \alpha(z)$ caractérise la diffusion des bulles. A(x) est la fonction auxiliaire cites plus hau
} 
. autres formes de diffuseurs (au moyen de l'introduction d'une matrice de diffusion non diagonale) - autres formes de nuages de diffuseurs,

. insonation bornée,

- matrice solide,

. ondes électromagnétiques ou optiques (peintures, SER radar etc.).

Le modèle permet le calcul des énergies transmises, une compréhension fine des mécanismes de diffusion multiple et enfin des comparaisons avec les résultats d'expérimentations calibrées.

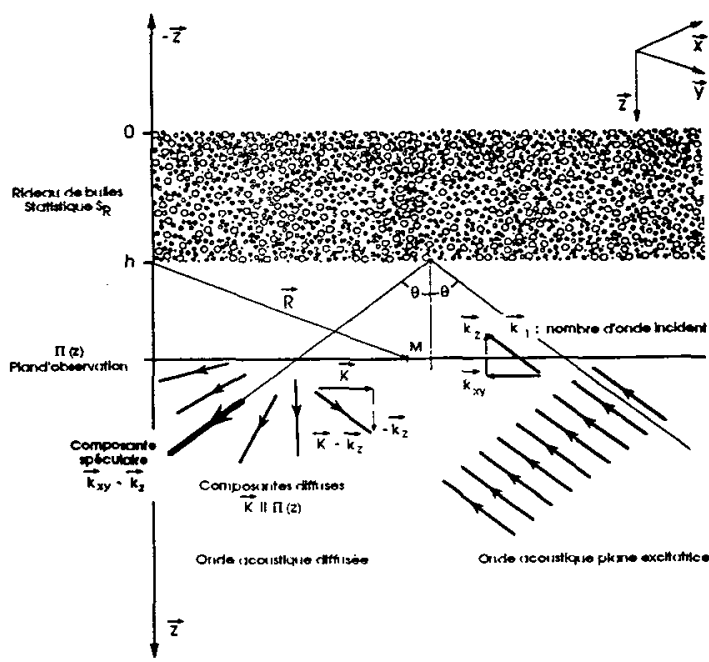

Figure 1 : configuration géométrique et notations

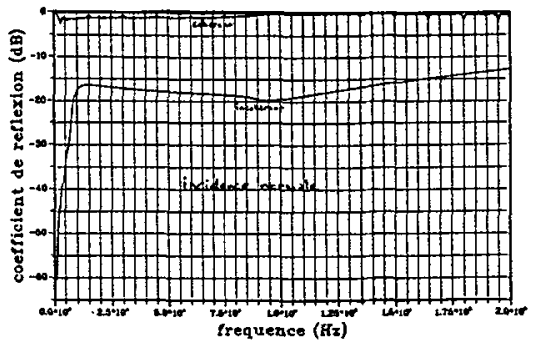

figure 4 : Variation fréquentielle des coefficients de rêflexion taux d'air $\sim 0,5 \%$. Statistique des rayons pour $30 \mu \leq a \leq 400 \mu p(a)=0,727 \exp (-0.03 a)$

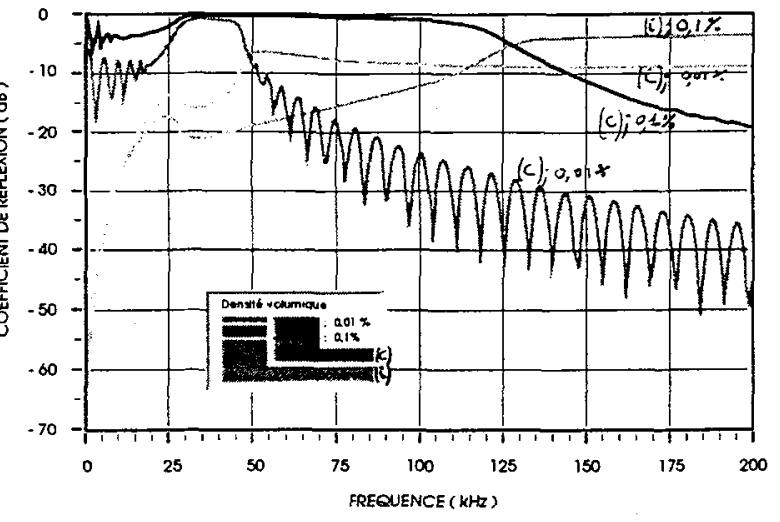

figure 2 : Variation fréquentielle des coefficients de réflexion cohérent et incohérent. Incidence normale. Bulles identiques $110 \mu \mathrm{m}$. densité $\beta$ variable.

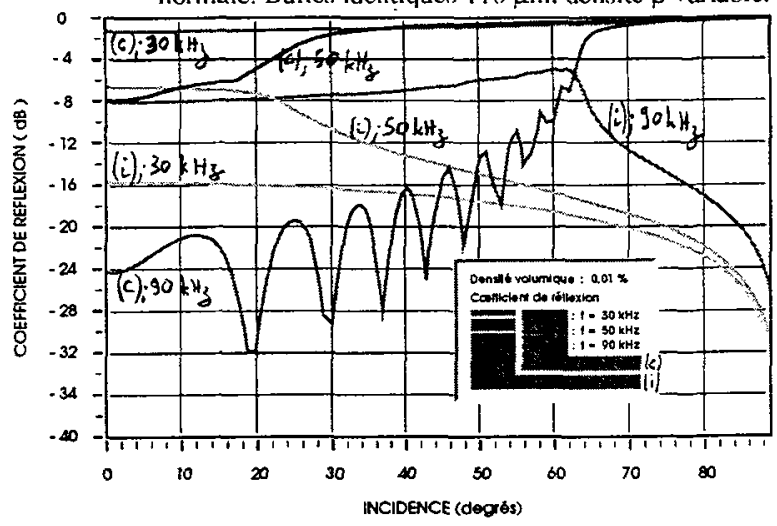

figure 3: Variation angulaire des coefficients de réflexion cohérent et incohérent. rayon identique $110 \mu$ taux d'air $0.01 \%$, fréquence variable.

\section{Références :}

[1] A. PROSPERETTI, L.A. CRUM. K.W. COMMANDER Non !inear bubble dynamics.JASA Vol. $83 n^{\circ} 2$ p. 502 (1988).

[2] A.J. RUDGERS : Analysis of thermoacoustic wave propagation in elastic media.JASA Vol. $88 \mathrm{n}^{\circ} 2$ p. 1078 (1990).

[3] Etude d'un revêtement de masquage à inclusions sphériques. Etude DRET/G6. marché 89/351. rapport final AERO PA/1692 (avril 1990 ).

[4] M. LAX : Multiple scattering of waves.

Reviews of Modern Physics. Vol. $23 \mathrm{n}^{\circ} 4$ p. 287 (1951).

[5] M. LAX : Multiple scattering of waves. II : the effective ficld in dense sysiems. Physical Review. Vol. $85 n^{\circ} 4$ p. 621 (1952).

[6] L.L. FOLDY.: The muitiple scattering of waves. I : General Theory of isotropic scattering by randomly distributed scatterers. Physical Review, Vol. $67 \mathrm{n}^{\circ} 3$ et 4 p. 107 (1945).

[7] Y. GERVAIS : La génération du bruit par conduction thermique. II Emission des sources multipolaires. doustica. Vol 70 p. 247 257 (1990).

[8] O. GERARD : Propagation acoustique dans un milieu diphasique eau-bulles d’air. Facteurs de réflexion er de transmission d'un rideau de bulles. Thèse de I'Université d'Aix-Marseille II - 1991.

[9] L. ROBIN : Fonctions sphériques de LEGENDRE et fonctions sphérö̈dales (tome 1, 2 et 3). GALUTHIER-VILLARS (1957).

[10] C.L RINO. H.D.NGO K.A. HAYCOCK : A spectral Domain method for multiple Scatering in discrete random media. IEEE Transaction on antennas and propagation vol $38 n^{\circ 7}$ Juillet 1990.

[11] C.L RINO. H.D.NGO : Low frequency acoustic seater from subsurface bubble elouds. J.ASA Vol $90 \mathrm{n}^{\circ} 1$ Juillet 1991. 